\title{
DETERMINACIÓN PROXIMAL DE LOS COMPONENTES NUTRICIONALES Y CARACTERIZACIÓN DEL TIPO DE ÁCIDOS GRASOS EN EL CONTENIDO LIPÍDICO DEL CATZO (Platycoelia lutescens)
}

\section{PROXIMAL DETERMINATION OF NUTRITIONAL COMPONENTS AND CHARACTERIZATION OF THE TYPE OF FATTY ACIDS IN THE LIPID CONTENT OF THE CATZO (Platycoelia lutescens)}

César Velastegui C., ${ }^{1}$ Katherine Batallas Z., ${ }^{1 *}$ Ana Hidalgo A. ${ }^{1}$ \& Eduardo Mayorga LI. ${ }^{1}$

Recibido: 28 de septiembre 2019 / Aceptado: 09 de diciembre 2019 DOI: 10.26807/ia.v8i1.120

Palabras clave: Cromatografía de gases, parámetros fisicoquímicos, perfil lipídico, Platycoelia lutescens, valor nutricional. Keywords: Gas chromatography, physicochemical parameters, lipidic profile, Platycoelia lutescens, nutritional value.

\section{RESUMEN}

Se reporta el valor nutricional y caracterización del contenido lipídico del catzo de la especie Platycoelia lutescen, recolectado en los cantones Cayambe y

1 Universidad Central del Ecuador, Facultad de Ciencias Químicas, Carrera Química de Alimentos, Quito, Ecuador (cgvelastegui@uce.edu.ec; *correspondencia: knbatallas@uce.edu.ec; amhidalgo@uce.edu.ec; emayorga@uce.edu.ec). 
Pedro Moncayo de la Provincia de Pichincha-Ecuador. Las localidades donde se recolectaron las muestras fueron: Tocachi, Chaupiloma, Paquiestancia y Cubinche; zonas donde tradicionalmente se prepara, consume y comercializa dicho escarabajo. Durante los meses de octubre, noviembre y diciembre, el mencionado escarabajo comestible de la región interandina, presenta su mayor pico poblacional del año, esto debido a que en el invierno esta especie endémica emerge a la superficie para reproducirse y es entonces cuando son recolectados. Para alcanzar el propósito metodológico de la presente investigación se prepararon previamente las muestras recolectadas, separando extremidades y alas debido a la forma de consumo del escarabajo. Se analizaron los parámetros fisicoquímicos de: humedad, cenizas, carbohidratos, proteína y grasa, llevándose a cabo una extracción de la fracción lipídica. Dentro de la caracterización de la grasa extraída se realizó el perfil lipídico mediante cromatografía de gases. Se pudo determinar que la especie Platycoelia lutescens presentó un contenido importante de proteína y grasa en las cuatro localidades analizadas, el cual difiere significativamente entre cada localidad; mientras que, parámetros fisicoquímicos como humedad y cenizas presentaron valores similares entre las localidades. En el contenido lipídico, se determinó presencia de ácidos grasos insaturados (Omega-6 y 9) con diferencia significativa entre localidades.

\section{ABSTRACT}

The purpose of this research project was to determine the nutritional value and characterize the lipid content of the catzo of the species Platycoelia lutescens collected in Cayambe and Pedro Moncayo cantons in the Pichincha Province of Ecuador. The 4 locations where the samples were collected are: Tocachi, Chaupiloma, Paquiestancia, Cubinche; areas where the beetle is traditionally prepared, consumed and marketed. During the months of October, November and December, the considered edible beetle of the inter-Andean region presents its highest population peak of the year, in these winter months this endemic species emerges to the surface to reproduce and local collect them. In order to achieve the methodological purpose of the present investigation, previously collected samples were prepared, separating limbs and wings because this is the form of consumption of the beetle; the physicochemical parameters of: humi- 
dity, ash, carbohydrates, protein and fat were analyzed and an extraction of the lipid fraction was carried out. Within the characterization of the extracted fat, the lipid profile was made by gas chromatography. It was determined that the species Platycoelia lutescens has an important content of protein and fat in the four localities analyzed, and this content differs significantly between each locality; Physicochemical parameters such as humidity and ash presented similar values between each locality, while in the lipid content, presence of unsaturated fatty acids (omega 6 and 9) was determined with significant difference between each locality.

\section{INTRODUCCIÓN}

En la actualidad, la nutrición se ha convertido en un tema de salud pública con gran importancia a nivel mundial. Conocer qué alimentos son indispensables en una dieta equilibrada y cuáles pueden brindar un valor agregado para la salud son aspectos que el consumidor toma en cuenta antes de hacer una elección; de aquí radica la necesidad de conocer la composición cualitativa y cuantitativa de todos los alimentos. En este contexto, determinar el valor nutricional de posibles fuentes alimenticias naturales e innovadoras es un paso fundamental para cumplir con el objetivo de promover la correcta nutrición de la población.
El análisis de parámetros nutricionales y explotar de manera responsable recursos naturales, como potencial fuente de alimento, es una forma de hacerle frente a problemáticas como la escasez de alimentos, sobrepeso, mal nutrición y desnutrición, males que aquejan a un gran porcentaje de la población en el país y que han sido muy difíciles de erradicar, sobre todo en zonas rurales y marginadas de la serranía ecuatoriana.

En el Ecuador, de acuerdo con datos de la Encuesta Nacional de Salud y Nutrición (2011-2013), uno de cada cuatro niños (menores de cinco años) padece de desnutrición crónica, pro- 
blema que se acentúa en las niñas. En la misma fuente se indica que en la población escolar de 5 a 11 años las prevalencias de desnutrición crónica están alrededor del $15 \%$, mientras que el sobrepeso llega al $32 \%$. Con relación a los adolescentes (12 a 19 años), en quienes persiste el retardo en talla, la obesidad rodea el $26 \%$, mientras que en los adultos el $63 \%$ tienen problemas de sobrepeso (ENSANUT, 2011- 2013).

La entomofagia, es el consumo de insectos por los seres humanos y es una práctica que se ha llevado a cabo en muchos países de todo el mundo, principalmente en regiones de Asia, África y Latinoamérica. La ingesta de insectos complementa la dieta de aproximadamente 2000 millones de personas, y se trata de un hábito que siempre ha estado presente en la conducta alimentaria de los seres humanos.

Los insectos están en todas partes, se reproducen rápidamente y poseen tasas elevadas de crecimiento, además de un reducido impacto ambiental durante su ciclo de vida. Son nutritivos, ya que contienen niveles elevados de proteínas, grasas y minerales. Pueden criarse aprovechando diversos flujos de residuos, como los residuos alimenticios. A nivel mundial, se consumen más de 1900 especies de insectos comestibles, de los cuales el mayor porcentaje de consumidos son los escarabajos (coleópteros), con un (31\%) el porcentaje restante se divide entre 8 diferentes especies y sub grupos de otros órdenes (FAO, 2013).

El Catzo blanco (Platycoelia lutescens) es una especie de escarabajo que se encuentra en las tierras altas de Colombia, Ecuador y Perú. Esta especie se reconoce fácilmente en relación con otros escarabajos andinos por el color crema y de gran tamaño, $2 \mathrm{~cm}$ aproximadamente (Smith \& Paucar, 2000).

En el país existe un sinnúmero de tradiciones culinarias aún no explotadas o que no han tenido el realce necesario; un claro ejemplo de esto se da en los cantones Cayambe y Pedro Moncayo. En estas zonas y en gran parte de la serranía ecuatoriana existe la tradición de recolectar, comercializar y consumir el catzo blanco el cual ha servido como fuente alimenticia de pobladores y visitantes por generaciones. 
En el 2003 la Escuela Politécnica del Ejército-Facultad de Ciencias Agropecuarias (IASA), realizó un análisis de los principios inmediatos del catzo blanco (Leucopelea albencens, Insecta: Coleoptera) obteniendo resultados halagadores en cuanto al contenido de proteínas, 27,11\%; valores que a su vez fueron comparados con los de otras especies animales consumidas como pavo y res, llegando a la conclusión de que el consumo de esta especie sugiere un aporte proteico significativo y es comparable con cualquier otra especie de cárnico. En cuanto al contenido lipídico, del mismo estudio se obtuvieron resultados de grasa total; sin embargo, no se reporta el contenido lipídico de la misma, dato que sería de gran importancia para la caracterización de esta grasa (Chiliquinga \& Pozo, 2003).

Basado en los antecedentes anteriores, en la presente investigación se reporta la determinación proximal de los componentes nutricionales y caracterización del tipo de ácidos grasos en el contenido lipídico del catzo (Platycoelia lutescens) recolectado en los cantones Cayambe y Pedro Moncayo (Tocachi, Chaupiloma, Paquiestancia, Cubinche) de la Provincia de Pichincha-Ecuador, a fin de establecer si existe diferencia en el valor nutricional y perfil lipídico de las muestras recolectadas en las diferentes localidades.

\section{MATERIALES Y MÉTODOS}

\section{Población}

La población que fundamentó la investigación fue el grupo de catzos blancos de especie Platycoelia lutescens recolectados en 4 zonas de los cantones Cayambe y Pedro Moncayo de la Provincia de Pichincha-Ecuador.

\section{Muestra}

Se realizó un muestreo probabilístico aleatorio simple, que consistió en recolectar, en 3 días diferentes, 500 gramos de catzo como muestra por cada localidad (Tocachi, Chaupiloma, Paquiestancia y Cubinche). Se formalizaron tres réplicas por cada muestra, tomando en cuenta la disponibilidad 
de catzos blancos, de especie Platycoelia lutescens, durante los meses de noviembre y diciembre de 2017; tiempo de abundancia por proceso reproductivo.

\section{Contenido total de proteínas}

El contenido de nitrógeno fue determinado usando el método oficial AOAC 981.10. El análisis realizado utilizó un Destilador Kjeldahl electrónico marca Velp Scientifica Digital, modelo UDK126A, serie 398017.

\section{Contenido total de grasa}

La determinación de grasas empleó el método oficial AOAC 991.36, basado en una extracción semicontínua Soxhlet.

\section{Humedad}

Análisis de humedad basado en el método oficial AOAC 925.10, utilizando una estufa eléctrica modelo 4 , Thelco Precesio.

\section{Contenido de cenizas totales}

Se determinó cenizas totales a través del Método Oficial AOAC 923.03. Se utilizó una mufla marca eléctrica, modelo 1400, Thermolyne.

\section{Contenido y tipo de ácidos grasos}

\section{Método de metilación}

El método que se utilizó para la metilación de ácidos grasos, previo al análisis cromatográfico, fue de acuerdo al Método Oficial AOAC 969.33.

\section{Análisis cromatográfico}

Se utilizó un equipo de Cromatografía de Gases YL Instrument 6500GC System, acoplado a un detector de ionización de llama, con una columna capilar de Fase reversa 60 metros de longitud por 0,25 milímetros de diámetro interno SELECTA para FAME y 0,2 $\mu \mathrm{m}$ de espesor de fase. Todo lo anterior de acuerdo al Método Oficial AOAC 996.06 para análisis cromatográfico de grasas en alimentos. La identificación de los ésteres metílicos de ácidos grasos de las muestras lipídicas de las cuatro localidades se realizó por comparación con los tiempos de retención del estándar F.A.M.E. Mix, C4-C24 SigmaAldrich. 


\section{Diseño experimental}

El diseño experimental en el cual se fundamentó este artículo es unifactorial completamente al azar con tres réplicas, lo que permite establecer la existencia de efectos en las variables elegidas. Para determinar si el valor nutricional y el contenido y tipo de ácidos grasos del catzo de especie
Platycoelia lutescens recolectado en los cantones Pedro Moncayo y Cayambe es diferente en cada una de las localidades de recolección, se realizó un análisis de varianza (ANOVA) y para determinar la diferencia entre los resultados obtenidos por las muestras recolectadas en cada parroquia se realizó la prueba de Tukey al $95 \%$ de confianza.

\section{RESULTADOS}

Análisis del valor nutricional del catzo Platycoelia lutescens.

En la Tabla 1, se presenta de forma sintetizada el contenido en porcen- taje de humedad, ceniza, proteína, grasa y carbohidrato de la especie de catzo Platycoelia lutescens de las localidades de Cayambe y Pedro Moncayo.

Tabla 1. Análisis proximal de la especie de catzo Platycoelia lutescens en localidades de Cayambe y Pedro Moncayo

\begin{tabular}{lcccc}
\hline \multirow{2}{*}{$\begin{array}{c}\text { Parámetro } \\
\text { \% }\end{array}$} & Tocachi & Chaupiloma & Paquiestancia & Cubinche \\
\cline { 2 - 5 } & $36,51 \pm 0,14$ & $35,68 \pm 0,49$ & $36,55 \pm 0,53$ & $35,43 \pm 0,04$ \\
Ceniza & $1,64 \pm 0,01$ & $1,65 \pm 0,03$ & $1,68 \pm 0,02$ & $1,58 \pm 0,04$ \\
Proteína & $24,69 \pm 0,08$ & $23,19 \pm 0,07$ & $26,08 \pm 0,18$ & $23,34 \pm 0,03$ \\
Grasa & $10,66 \pm 0,30$ & $14,19 \pm 0,03$ & $11,74 \pm 0,11$ & $9,62 \pm 0,28$ \\
Carbohidrato & $26,50 \pm 0,28$ & $25,30 \pm 0,41$ & $23,95 \pm 0,30$ & $30,02 \pm 0,30$ \\
\hline
\end{tabular}


Contenido de ácidos grasos de la especie de catzo blanco Platycoelia lutescens.

Los extractos de grasa obtenidos de las muestras de catzos fueron tratados para obtener los ésteres metílicos de ácidos grasos y por medio de cromatografía de gases se logró identificar los tipos de ácidos grasos presentes en la grasa y sus concentraciones.La Figura 1 representa un cromatograma con el reporte de los principales ácidos grasos identificados en una muestra de catzo blanco de la localidad de Tocachi.

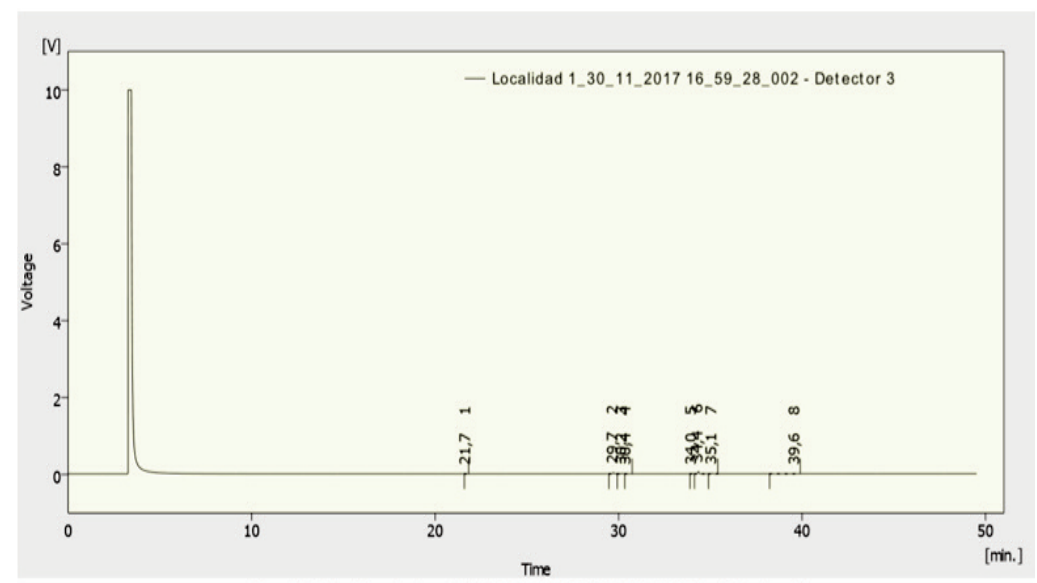

Result Table (Uncal - Localidad 1_30_11_2017 16_59_28_002 - Detector 3)

\begin{tabular}{|r|r|r|r|r|r|r|l|}
\hline & $\begin{array}{c}\text { Reten. Time } \\
{[\mathrm{min}]}\end{array}$ & \multicolumn{1}{c|}{$\begin{array}{c}\text { Area } \\
{[\mathrm{mV} . \mathrm{s}]}\end{array}$} & \multicolumn{1}{c|}{$\begin{array}{c}\text { Heght } \\
{[\mathrm{mV}]}\end{array}$} & \multicolumn{1}{c|}{$\begin{array}{c}\text { Area } \\
{[\%]}\end{array}$} & \multicolumn{1}{c|}{$\begin{array}{c}\text { Heght } \\
{[\%]}\end{array}$} & \multicolumn{1}{c|}{$\begin{array}{c}\text { W05 } \\
{[\mathrm{min}]}\end{array}$} & \multicolumn{1}{c|}{$\begin{array}{c}\text { Compound } \\
\text { Name }\end{array}$} \\
\hline 1 & 21,683 & 7,390 & 1,576 & 1,2 & 1,3 & 0,07 & Acido Tridecanoico \\
\hline 2 & 29,717 & 148,442 & 32,282 & 24,5 & 25,7 & 0,07 & Acido Palmítico \\
\hline 3 & 30,183 & 64,708 & 13,530 & 10,7 & 10,8 & 0,07 & Acido Paimitoleico \\
\hline 4 & 30,427 & 13,37 & 2,604 & 2,2 & 2,1 & 0,08 & \\
\hline 5 & 34,007 & 22,542 & 4,844 & 3,7 & 3,9 & 0,08 & Acido Estearico \\
\hline 6 & 34,353 & 284,571 & 59,346 & 47,0 & 47,3 & 0,08 & Acido Oleico \\
\hline 7 & 35,100 & 48,210 & 10,27 & 8,0 & 8,2 & 0,08 & Acido linoleico (omeza 6) \\
\hline 8 & 39,627 & 16,433 & 1,090 & 2,7 & 0,9 & 0,09 & Acido Cis-11,14-Eicosadienoico \\
\hline & Total & 605,673 & 125,548 & 100,0 & 100,0 & & \\
\hline
\end{tabular}

Figura 1. Cromatograma de ácidos grasos de catzo blanco muestra L1: Tocachi 
En la Tabla 2 se detalla el contenido de todos los ácidos grasos presentes en las muestras de la especie de catzo blanco Platycoelia lutescens recolectado en las localidades de Tocachi, Chaupiloma, Paquiestancia y
Cubinche. La Figura 2 compara el contenido y el tipo de ácidos grasos siendo los más representativos el ácido palmítico, ácido palmitoleico, ácido oleico y ácido linoleico.

\section{Tabla 2. Contenido de ácidos grasos de la especie de catzo blanco Platycoelia lutescens}

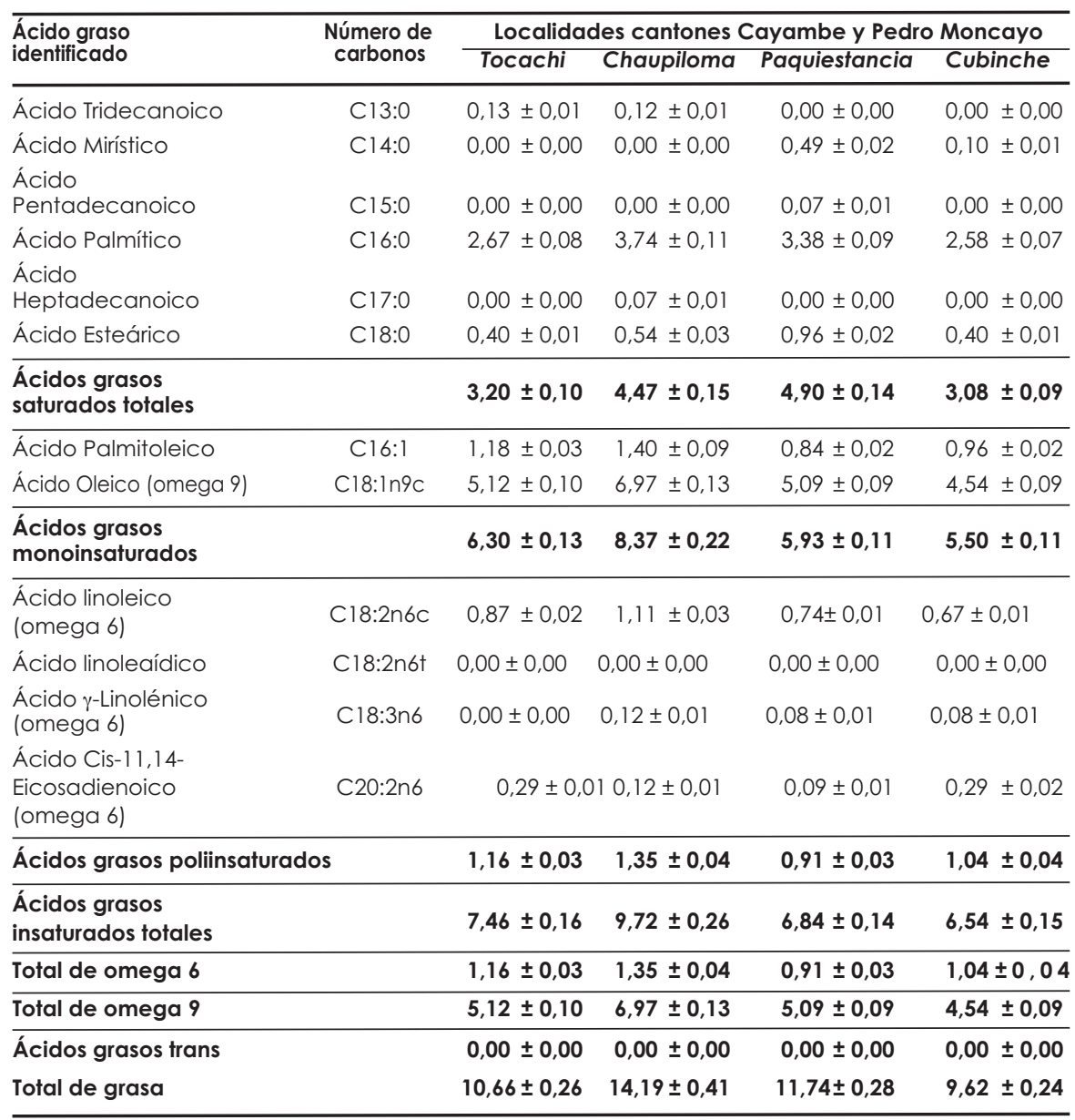




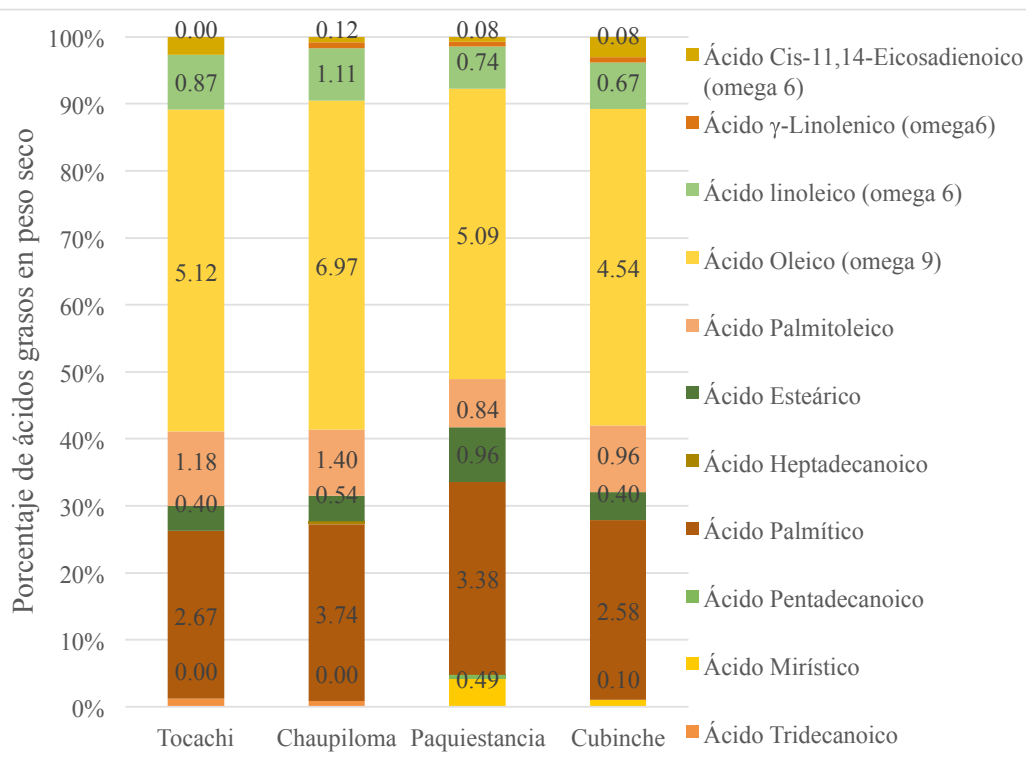

Localidades de los cantones: Cayambre y Pedro Moncayo

Figura 2. Comparación del tipo de ácidos grasos en la especie de catzo blanco Platycoelia lutescens de las localidades de los cantones Cayambe y Pedro Moncayo

\section{DISCUSIÓN}

Para el parámetro proteína las muestras recolectadas en la localidad de Paquiestancia presentan el mayor porcentaje de proteína con un valor de $26,08 \% \pm 0,18$. Estos resultados demuestran el aporte proteico que tiene el catzo cuando es utilizado como alimento, considerando que las proteínas interfieren en la formación de tejidos y músculos, se con- vierte en un alimento con aporte nutricional.

En el análisis del contenido lipídico se determinó que existe diferencia entre todos los valores obtenidos de porcentaje de grasa en las 4 localidades, siendo las muestras recolectadas en la localidad de Chaupiloma aqueIlas que presentaron el mayor por- 
DETERMINACIÓN PROXIMAL DE LOS COMPONENTES NUTRICIONALES Y CARACTERIZACIÓN DEL TIPO DE ÁCIDOS GRASOS EN EL CONTENIDO LIPÍDICO DEL CATZO (Platycoelia lutescens)

Velasteguí et. al.,113-125

centaje de grasa con un valor de $14,19 \% \pm 0,03$. Santurino et. al. (2016), en un estudio sobre los insectos como complemento nutricional de la dieta: fuente de lípidos potencialmente activos, los investigadores analizan el contenido de grasa de cuatro especies de insectos consumidos en Madrid España entre estos grillos común, langosta migratoria, gusano de la harina y gusano rey, determinando los contenidos de grasa de cada especie, y siendo el insecto Grillo común Acheta domesticus $15,25 \%$ de grasa el que mayor similitud en cuanto al contenido de grasa presenta con los catzos de especie Platycoelia lutescens.

Entre los ácidos grasos identificados en el componente lipídico de catzos de especie Platycoelia lutescens se destacan el ácido oleico como el principal constituyente que pertenece al grupo de ácidos grasos insaturados y se lo ubica en el grupo de Omega-9, el segundo tipo de ácido graso más abundante en la grasa del catzo de especie Platycoelia lutescens es el ácido palmítico, este ácido graso corresponde al grupo de ácidos grasos saturados característicos de la composición lipídica de la grasa de animales e insectos.
Entre los ácidos grasos Omega-6 encontrados se encuentran el ácido linoleico y el ácido eicosadienoico cuyas concentraciones son similares en los catzos de las cuatro localidades analizadas. Molina \& Martín, (2010), en su estudio ácidos grasos esenciales Omega-3 y Omega-6 establecen la importancia del consumo de estos ácidos grasos en la dieta de los seres humanos, ya que los ácidos Omega-6 y Omega-9 interfieren en algunas funciones metabólicas del cuerpo humano, actuando como precursores de otro tipo de compuestos, por ejemplo el ácido linoleico puede dar origen al ácido araquidónico con importantes funciones metabólicas y reguladoras formando parte de la estructura de los fosfolípidos de las membranas celulares particularmente la fosfatidilcolina, fosfatidiletanolamina y fosfatidilserina.

En el análisis del tipo de ácidos grasos, no se encontró ningún tipo de ácido graso trans. La Organización Mundial de la Salud (OMS 2018) en su guía Replace, determina lo nocivo que son los ácidos grasos trans en el consumo en alimentos, y plantea la importancia de que estos ácidos grasos no sean introducidos en la dieta para evitar enfermedades cardiovasculares. 


\section{CONCLUSIONES}

Se determinó el valor nutricional y caracterizó el tipo de ácidos grasos en el contenido lipídico del catzo de especie Platycoelia lutescens recolectado en los cantones Cayambe y Pedro Moncayo de la Provincia de Pichincha-Ecuador, lugares donde este insecto es consumido y es parte de la dieta de los habitantes del sector.

Los resultados determinan valores representativos en el contenido de proteína del catzo Platycoelia lutescens comparable con alimentos de origen vegetal y animal. Sería muy importante realizar estudios sobre los tipos de aminoácidos que contiene esta proteína.
Se caracterizó el contenido lipídico y cuantificó los ácidos grasos del catzo Platycoelia lutescens mediante la técnica de cromatografía de gases. Los ácidos grasos insaturados que se encontraron fueron: ácido palmitoleico, ácido oleico (Omega-9), ácido linoleico (Omega-6), ácido $\gamma$-Linolénico (Omega-6) y ácido cis-11,14-eicosadienoico (Omega-6). Además, en base a los resultados obtenidos se determinó que en ninguna de las muestras recolectadas hubo presencia de grasas trans en el contenido lipídico extraído. 


\section{LISTA DE REFERENCIAS}

AOAC. (2000). Official Methods of Analysis. Gaithersburg: Association of Official Analytical Chemists International.

AOCS. (1997). Determinación de los ácidos grasos. Métodos de la Sociedad Americana de Químicos de Aceites.

Chiliquinga, M. I., \& Pozo, W. (2003). Calidad nutritiva del Catzo Blanco. IASA de la Escuela Politéecnica del Ejercito, 55-57.

ENSANUT. (2011- 2013). Encuesta Nacional de Salud y Nutrición. Quito, Ecuador: Ministerio de salud pública, INEC.

FAO. (2013). La contribución de los insectos a la seguridad alimentaria, los medios de vida y el medio ambiente. Roma.: Organización de las Naciones Unidas para la Alimentación y la Agricultura. Obtenido de http://www.fao.org/3/i3264s/ i3264s00.pdf

Montes, M. (2010). Ácidos grasos esenciales Omega-3 Y Omega-6 Papel en el embarazo y la lactancia. Ámbito Farmacéutico, Nutrición., (29), 66-72.

Onore, G. (1997). A brief note on edible insects in Ecuador. Ecol. Food Nutr, 36:277285.

Santurino, C., García-Serrano, A., Molina García, J., Sierra, P., Castro-Gómez, M., \& Fontecha, J. (2016). Los insectos como complemento nutricional de la dieta: fuente de lípidos potencialmente bioactivos. Alimentación, nutrición y salud, (23), 6066.

Smith, A., \& Paucar, A. (2000). Taxonomic review of Platycoelia lutescens Blanchard (Scarabaeidae: Rutelinae: Anoplognathini) and a description of the use of this species as food by the people of the Ecuadorian highlands. Lincoln: University of Nebraska State. 